\title{
THE ORDER OF A GROUP OF EVEN ORDER
}

\author{
HIROYOSHI YAMAKI
}

(Communicated by Jonathan I. Hall)

\begin{abstract}
We will give an estimation of the order of a group of even order by the order of the centralizer of an involution using the classification of finite simple groups.
\end{abstract}

\section{INTRODUCTION}

Let $G$ be a group of even order and $t$ an involution in $G$. Brauer and Fowler [2] proved that if $G$ is a finite simple group of even order, then $|G|<\left(\left|C_{G}(t)\right|^{2}\right)$ !. It is well known that $|G|<\left|C_{G}(u)\right|^{3}$ for some involution $u$ in $G$ if $G$ has more than one conjugacy class of involutions (see Suzuki [13, p. 127]). It has been conjectured by $K$. Harada that if $G$ has precisely one conjugacy class of involutions and $G$ is not 2-rank 1, then $|G|<\left|C_{G}(u)\right|^{3}$ for an involution $u$ in $G$. The purpose of this note is to affirmatively prove Harada's conjecture using the classification of finite simple groups which is now complete. Namely we will prove:

Theorem 1. Let $G$ be a group of even order. Suppose that $G$ has precisely one conjugacy class of involutions and $G$ is not 2-rank 1 . Then $|G|<\left|C_{G}(t)\right|^{3}$ for an involution $t$ in $G$.

Combining a well known fact mentioned above we have:

Corollary 1. Let $G$ be a group of even order. Suppose that $G$ is not 2-rank 1. Then $|G|<\left|C_{G}(t)\right|^{3}$ for some involution $t$ in $G$.

Remark 1. Let $G$ be a finite group with 2-rank 1. Then the Sylow 2-subgroup of $G$ is cyclic or a generalized quaternion. Let $t$ be an involution in $G$. Then $G=C_{G}(t) O(G)$ by Burnside's or Brauer-Suzuki's theorem (see Suzuki [13, p. 144, p. 306]). If $C_{G}(t) \cap O(G)=1$ and $|O(G)|>\left|C_{G}(t)\right|^{2}$, then $|G|>\left|C_{G}(t)\right|^{3}$. Thus the conclusion of Theorem 1 does not hold true in general if $G$ is 2-rank 1 .

Remark 2. Let $\pi$ be the connected component of the prime graph of $G$ containing 2 (see Williams [14], Kondrat'ev [12], Iiyori-Yamaki [11]). Let $\Omega$ be the set of $\pi$ elements of $G$. For $v \in \Omega$ define $\operatorname{Inv}\left(C_{G}^{-1}(v)\right)=\left\{x \in G \mid x^{-1} v x=v^{-1}, x^{2}=1\right\}$. Recently Harada and Miyamoto [9] proved that if $G$ has precisely one conjugacy

Received by the editors August 15, 2006.

2000 Mathematics Subject Classification. Primary 20D05, 20 D06.

Key words and phrases. Finite simple groups, centralizers of involutions.

The author was supported in part by Grant-in-Aid for Scientific Research (No. 16540030), Japan Society for the Promotion of Science. 
class of involutions and the Sylow 2-subgroup of $G$ is not cyclic or a generalized quaternion, then $|G|<\left|C_{G}(t)\right|^{3}+m_{\pi}\left|C_{G}(t)\right|^{2}$ for an involution $t$ and for $m_{\pi}=\max \left\{\left|C_{G}(t)\right|,\left|\operatorname{Inv}\left(C_{G}^{-1}(v)\right)\right| ; v \in \Omega\right\}$ without using the classification of finite simple groups. Furthermore they showed that the orders of the sporadic simple groups $T h$ and $L y$ can easily be determined by those of the centralizers of involutions using their result.

Our notation is standard (see Atlas [5], Suzuki [13]).

\section{Finite Simple Groups}

The purpose of this section is to prove two lemmas on finite simple groups using the classification.

Lemma 1. Let $G$ be a finite simple group with precisely one conjugacy class of involutions. Then $|G|<\left|C_{G}(t)\right|^{3}$ for an involution $t$ in $G$.

Proof. Let $G$ be a non-abelian simple group with precisely one conjugacy class of involutions and $t$ an involution in $G$. It follows that $G$ is isomorphic to one of the groups for suitable $q$ :

$$
\begin{aligned}
& A_{5}, A_{6}, A_{7}, \\
& L_{2}(q), L_{3}(q), L_{4}(q), U_{3}(q), U_{4}(q), G_{2}(q),{ }^{2} G_{2}(q),{ }^{3} D_{4}\left(q^{3}\right), S z(q), \\
& M_{11}, M_{22}, M_{23}, J_{1}, J_{3}, M c L, O N, L y, T h .
\end{aligned}
$$

Then we have the following tables which are self-explanatory. We refer to Atlas [5] for the sporadic simple groups, Burgoyne-Williamson [3], Suzuki [13], Williams [14] for the simple groups of Lie type over the field of odd characteristic, and AschbacherSeitz [1], Suzuki [13], Dye [6, 7, 8] for those over the field of even characteristic. It follows from Tables 1-4 below that $|G|<\left|C_{G}(t)\right|^{3}$, which completes the proof.

Corollary 2. Let $G$ be a finite simple group. Then $|G|<\left|C_{G}(t)\right|^{3}$ for some involution $t$ in $G$.

Lemma 2. There exists no finite simple group $G$ such that $G$ has $k$ conjugacy classes of involutions for $k \geq 2$ and all involutions in $G$ are conjugate under Aut $(G)$.

TABLE 1. Alternating Groups

\begin{tabular}{|c|c|c|}
\hline$G$ & $\left|C_{G}(t)\right|$ & $|G|$ \\
\hline \hline$A_{5}=L_{2}(4)$ & $2^{2}$ & $60=2^{2} .3 .5$ \\
\hline$A_{6}=L_{2}\left(3^{2}\right)$ & $2^{3}$ & $360=2^{3} .3^{2} .5$ \\
\hline$A_{7}$ & $2^{3} .3$ & $2520=2^{3} .3^{2} .5 .7$ \\
\hline
\end{tabular}

TABLE 2. Groups of Lie type over the field of even characteristic

\begin{tabular}{|c|c|c|}
\hline$G$ & $\left|C_{G}(t)\right|$ & $|G|$ \\
\hline \hline$L_{2}(q)$ & $q$ & $(q+1) q(q-1)$ \\
\hline$L_{3}(q)$ & $q^{3}(q-1)(3, q-1)^{-1}$ & $\left(q^{3}-1\right)\left(q^{2}-1\right) q^{3}(3, q-1)^{-1}$ \\
\hline$U_{3}(q)$ & $q^{3}(q+1)(3, q+1)^{-1}$ & $\left(q^{3}+1\right) q^{3}\left(q^{2}-1\right)(3, q+1)^{-1}$ \\
\hline$S z(q), q=2^{2 k+1}, k \geq 1$ & $q^{2}$ & $\left(q^{2}+1\right) q^{2}(q-1)$ \\
\hline
\end{tabular}


TABLE 3. Groups of Lie type over the field of odd characteristic

\begin{tabular}{|c|c|c|}
\hline$G$ & $\left|C_{G}(t)\right|$ & $|G|$ \\
\hline \hline$L_{2}(q), q \equiv 1(4)$ & $q-1$ & $q\left(q^{2}-1\right) / 2$ \\
\hline$L_{2}(q), q \equiv-1(4)$ & $q+1$ & $q\left(q^{2}-1\right) / 2$ \\
\hline$L_{3}(q)$ & $(q-1)^{2} q(q+1)(3, q-1)^{-1}$ & $q^{3}\left(q^{2}-1\right)\left(q^{3}-1\right)(3, q-1)^{-1}$ \\
\hline$L_{4}(q), q \equiv 5(8)$ & $(q-1)^{3} q^{2}(q+1)^{2} / 2$ & $q^{6} \prod_{i=1}^{3}\left(q^{i+1}-1\right) / 4$ \\
\hline$G_{2}(q)$ & $q^{2}\left(q^{2}-1\right)^{2}$ & $q^{6}\left(q^{6}-1\right)\left(q^{2}-1\right)$ \\
\hline${ }^{2} G_{2}(q), q=3^{2 k+1}, k \geq 2$ & $q\left(q^{2}-1\right)$ & $q^{3}\left(q^{3}+1\right)(q-1)$ \\
\hline$U_{3}(q)$ & $q(q+1)\left(q^{2}-1\right)(3, q+1)^{-1}$ & $\left(q^{3}+1\right) q^{3}\left(q^{2}-1\right)(3, q+1)^{-1}$ \\
\hline$U_{4}(q), q \equiv 3(8)$ & $(q-1)^{2} q^{2}(q+1)^{3} / 2$ & $q^{6} \prod_{i=1}^{3}\left(q^{i+1}-(-1)^{i+1}\right) / 4$ \\
\hline$D_{4}\left(q^{3}\right)$ & $q^{4}\left(q^{2}-1\right)\left(q^{6}-1\right)$ & $q^{12}\left(q^{8}+q^{4}+1\right)\left(q^{6}-1\right)\left(q^{2}-1\right)$ \\
\hline
\end{tabular}

TABLE 4. Sporadic simple groups

\begin{tabular}{|c|c|c|}
\hline$G$ & $\left|C_{G}(t)\right|$ & $|G|$ \\
\hline 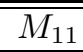 & $\overline{2^{4} .3}$ & $2^{4} .3^{2} .5 .11$ \\
\hline$J_{1}$ & $2^{3} .3 .5$ & $2^{3} .3 .5 .7 .11 .19$ \\
\hline$M_{22}$ & $2^{7} .3$ & $2^{7} \cdot 3^{2} \cdot 5.7 .11$ \\
\hline$M_{23}$ & $2^{7} .3 .7$ & $2^{7} \cdot 3^{2} \cdot 5 \cdot 7 \cdot 11.23$ \\
\hline$J_{3}$ & $2^{7} .3 .5$ & $2^{7} .3^{5} \cdot 5.17 .19$ \\
\hline$\overline{M c L}$ & $2^{7} .3^{2} \cdot 5.7$ & $2^{7} \cdot 3^{6} \cdot 5^{3} \cdot 7.11$ \\
\hline$O N$ & $2^{9} .3^{2} .5 .7$ & $2^{9} \cdot 3^{4} \cdot 5.7^{3} \cdot 11.19 .31$ \\
\hline$L y$ & $2^{8} .3^{4} \cdot 5^{2} .7 .11$ & $2^{8} .3^{7} .5^{6} .7 .11 .31 .37 .67$ \\
\hline$T h$ & $2^{15} \cdot 3^{4} \cdot 5.7$ & $2^{15} \cdot 3^{10} \cdot 5^{3} \cdot 7^{2} \cdot 13 \cdot 19.31$ \\
\hline
\end{tabular}

Proof. By our assumption all centralizers of involutions in $G$ are isomorphic, and in particular any involution is contained in the center of a Sylow 2-subgroup of $G$. It is almost trivial that this does not happen when $G$ is isomorphic to $A_{n}(n \geq 8)$ or one of the sporadic simple groups (see Atlas [5]). We assume that $G$ is a group of Lie type.

Case 1 . Let $G$ be a group of Lie type over the field of odd characteristic. Then $G$ is a group of component type. We look at the components of $G$ to know whether the centralizers of involutions are isomorphic or not (see Burgoyne-Williamson [3] or Williams [14]). It will be easily seen that the centralizers of involutions in $G$ are not isomorphic.

Let $G=L_{n}(q), n-2=r+s, r>0, s>0$ and $d=(n, q-1)$. If $n$ is odd, $G$ contains centralizers of type $A_{r} \times A_{s}$. It follows that $n=3$ and $k=1$, a contradiction. Suppose that $n$ is even. If $s$ is even or $q \equiv 1(2 d)$, then there exist centralizers of type $A_{r} \times A_{s}$. If $r=s$ and $q \not \equiv 1(2 d)$, then there is a centralizer of type $A_{r}\left(q^{2}\right)$. These centralizers of involutions are not isomorphic. This proves our lemma for $G=L_{n}(q)$.

Let $G=U_{n}(q), n-2=r+s$ and $t$ a 2-part of $n$. If $n$ is odd, then $t=1$ and there are centralizers of type ${ }^{2} A_{r} \times{ }^{2} A_{s}$ for $0 \leq r \leq(n-1) / 2$. It follows that $n=3$ and $k=1$. This is not our case. Suppose that $n$ is even. If $q \equiv-1(2 t)$, there are centralizers of type ${ }^{2} A_{r} \times{ }^{2} A_{s}$ for $0 \leq r \leq(n-2) / 2$. If $q \equiv 1(2 t)$, there are 
centralizers of type ${ }^{2} A_{r} \times{ }^{2} A_{s}$ for $r$ odd, $0 \leq r \leq(n-2) / 2$ and of type ${ }^{2} A_{(n-2) / 2}$. They are not isomorphic.

Let $G=S_{2 m}(q)$ and $m=r+s, r>0, s>0$. If $r \neq s$ or $r=s$ is even, then there exist centralizers of type $C_{r} \times C_{s}$. Assume $r=s$. Then $G$ contains a centralizer of type $A_{m-1}$ if $q \equiv 1(4)$ or of type ${ }^{2} A_{m-1}$ if $q \equiv-1(4)$. This proves our lemma for $S_{2 m}(q)$.

Let $G=O_{2 n+1}(q)$ and $r+s=n, 0 \leq r \leq n-1,2 \leq s \leq n . G$ contains a centralizer of type $B_{n-1}$. If $q \equiv 1(4)$, there exist centralizers of type $B_{r} \times D_{s}$. If $q \equiv-1(4)$, then there exist centralizers of type $B_{r} \times D_{s}$ when $s$ is even or of type $B_{r} \times{ }^{2} D_{s}$ when $s$ is odd. This proves our lemma for $G$ when $n \geq 3$.

Let $G=O_{8}^{+}(q)$. Then $G$ contains a centralizer of type $D_{2} \times D_{2}$. There exists a centralizer of type $A_{3}$ if $q \equiv 1(4)$ and type ${ }^{2} A_{3}$ if $q \equiv-1(4)$. They are not isomorphic.

Let $G=O_{8}^{-}(q)$. Then $G$ contains a centralizer of type $D_{2} \times{ }^{2} D_{2}$. There exists a centralizer of type ${ }^{2} A_{3}$ if $q \equiv 1(4)$ and of type $A_{3}$ if $q \equiv-1(4)$. They are not isomorphic.

Let $G=O_{2 n}^{+}(q)$. Suppose that $n$ is even and $n \geq 6$. There exist centralizers of type $D_{r} \times D_{s}$ where $r$ is even and $r+s=n, 2 \leq r \leq(n-2) / 2$. If $q \equiv 1(4)$ or $n$ is divisible by 4 , then there exists a centralizer of type $D_{n / 2} \times D_{n / 2}$. If $q \equiv-1(4)$, then $G$ contains a centralizer of type ${ }^{2} D_{n / 2} \times{ }^{2} D_{n / 2}$. They are not isomorphic. Suppose that $n$ is odd and $n \geq 5$. There exist centralizers of type $D_{n-1}$ and of type $D_{r} \times D_{s}, r+s=n, 2 \leq r \leq(n+1) / 2$ for $r$ even. They are not isomorphic.

Let $G=O_{2 n}^{-}(q)$. Suppose that $n$ is even and $n \geq 6$. There exist centralizers of type $D_{n-1}$ and of type ${ }^{2} D_{n-1}$. They are not isomorphic. Suppose that $n$ is odd and $n \geq 5$. There exist centralizers of type $D_{n-1}$ and of type $D_{r} \times{ }^{2} D_{s}$ where $r$ is even and $r+s=n, 2 \leq r \leq(n+1) / 2$. They are not isomorphic.

Let $G=E_{6}(q)$. There exist centralizers of type $D_{5}$ and of type $A_{1} \times A_{5}$.

Let $G={ }^{2} E_{6}(q)$. There exist centralizers of type ${ }^{2} D_{5}$ and of type $A_{1} \times{ }^{2} A_{5}$.

Let $G=E_{7}(q)$. Then $G$ contains a centralizer of type $A_{1} \times D_{6}$. If $q \equiv 1(4)$, then there exist centralizers of type $E_{6}$ and of type $A_{7}$. If $q \equiv-1(4)$, then there exist centralizers of type ${ }^{2} E_{6}$ and of type ${ }^{2} A_{7}$.

Let $G=E_{8}(q)$. There exist centralizers of type $D_{8}$ and $A_{1} \times E_{7}$.

Let $G=F_{4}(q)$. There exist centralizers of type $B_{4}$ and of type ${ }^{2} A_{7}$.

We note that the groups ${ }^{3} D_{4}(q), G_{2}(q),{ }^{2} G_{2}(q)$ have a unique conjugacy class of involutions when $q$ is odd.

Case 2. Let $G$ be a group of Lie type over the field of even characteristic. If $G$ is a classical simple group and $G$ is not $S_{4}(q)$, then $G$ contains a non-central involution, which is not our case (see Dye $[6,7,8]$ ). If $G=S_{4}(q), q$ even, then $G$ has three conjugacy classes of involutions and all of them are central. Two of them have the same cardinalities but the third one is different from the other two. If $G$ is an exceptional group of rank 2 , then $G$ has two conjugacy classes of involutions and one of them is non-central. If $G$ is an exceptional group of rank greater than 2 , then it follows from Aschbacher-Seitz [1] that we have Table 5 of $C_{G}(t) / O_{2}\left(C_{G}(t)\right)$ for an involution $t \in G$ (see also Chigira-Iiyori-Yamaki [4], Kondrat'ev [12]) which eventually yields our lemma for $G$. We note that $F_{4}(q)$ has four conjugacy classes of involutions, and two of them are fused by the graph automorphism. 
TABLE 5

\begin{tabular}{|c|c|}
\hline$G$ & $C_{G}(t) / O_{2}\left(C_{G}(t)\right)$ \\
\hline \hline \multirow{3}{*}{$F_{4}(q)$} & $S_{6}(q)$ \\
& $S_{6}(q)$ \\
& $S_{4}(q)$ \\
\hline \multirow{2}{*}{$E_{6}(q)$} & $S L(2, q) \times S L(2, q)$ \\
& $S U(6, q)$ \\
& $(S L(2, q) \times S L(2, q)): Z_{q+1}$ \\
\hline \multirow{3}{*}{$E_{6}(q)$} & $S L(6, q)$ \\
& $S_{6}(q) \times Z_{q-1 /(3 . q-1)}$ \\
\hline & $S L(2, q) \times S L(3, q)$ \\
\hline \multirow{5}{*}{$E_{7}(q)$} & $S O^{+}(12, q)$ \\
& $S L(2, q) \times S_{6}(q)$ \\
& $F_{4}(q)$ \\
& $S_{6}(q)$ \\
\hline \multirow{2}{*}{$E_{8}(q)$} & $E_{7}(q)$ \\
& $S_{12}(q)$ \\
& $F_{4}(q) \times S L(2, q)$ \\
& $S_{8}(q)$ \\
\hline
\end{tabular}

\section{The Proof of TheOrem 1}

Let $G$ be a minimal counterexample to Theorem 1. Suppose that $G$ is not simple. Let $N$ be a minimal normal subgroup of $G$. Then $N$ is an elementary abelian $p$-group or a direct product of isomorphic non-abelian simple groups.

Lemma 3. Suppose that $N$ is an elementary abelian 2-group. Then Theorem 1 holds true for $G$.

Proof. Let $t \in N$ be an involution. Since $G$ has one conjugacy class of involutions, $|N|-1=\left(G: C_{G}(t)\right)$ and $C_{G}(t) \supseteq N$. It follows that $|G|<\left(\left|C_{G}(t)\right|-1\right)\left|C_{G}(t)\right|<$ $\left|C_{G}(t)\right|^{3}$.

Lemma 4. Suppose that $N$ is an elementary abelian p-group for odd prime $p$. Then Theorem 1 holds true for $G$.

Proof. The group $G / N$ satisfies the condition of Theorem 1. Let $t$ be an involution in $G$. Then by induction $\left|C_{G / N}(t N)\right|^{3}>|G / N|$. Note that $C_{G}(t) N / N=$ $C_{G / N}(t N)$. Let $\langle t, u\rangle$ be a four group in $G$. Since $t \sim u \sim t u$ in $G$, BrauerWielandt's formula (see Huppert-Blackburn [10, p. 290]) implies that

$$
|N|=\left|C_{N}(t)\right|\left|C_{N}(u)\right|\left|C_{N}(t u)\right| /\left|C_{N}(\langle t, u\rangle)\right|^{2}=\left|C_{N}(t)\right|^{3} /\left|C_{N}(\langle t, u\rangle)\right|^{2} .
$$

Since $\left|C_{G}(t) N\right|^{3} /|N|^{3}>|G| /|N|$ it follows that $\left|C_{G}(t) N\right|^{3}>|G||N|^{2}$. This yields

$$
\left|C_{G}(t)\right|^{3} \geq\left|C_{G}(t)\right|^{3} /\left|C_{N}(\langle t, u\rangle)\right|^{2}>|G|
$$

which completes the proof.

Lemma 5. Suppose that $N=S \times S \times S \times \cdots \times S$ where $S$ is a non-abelian simple group. Then $N=S$ and Theorem 1 holds true for $G$.

Proof. Since $G$ has one conjugacy class of involutions it is easy to see that $N=S$. Let $t \in S$ be an involution. If $S$ has one conjugacy class of involutions, then 
$\left(S: C_{S}(t)\right)=\left(G: C_{G}(t)\right)$. Since $\left|C_{S}(t)\right|^{3}>|S|$ by Lemma 1, it follows that $\left|C_{G}(t)\right|^{3}>|G|$. Suppose that $S$ has $k(\geq 2)$ conjugacy classes of involutions. Since $G$ has precisely one conjugacy class of involutions, all of the centralizers of involutions in $S$ are isomorphic to $C_{S}(t)$. Since $t$ is a central involution in a Sylow 2-subgroup of $G$, it follows that $\left(G: C_{G}(t)\right)=k\left(S: C_{S}(t)\right)$ and $k$ is odd. This does not happen in finite simple groups by Lemma 2 .

By Lemmas 3, 4 and 5 we may assume that $G$ is a non-abelian simple group. It follows from Lemma 1 that Theorem 1 holds true for $G$. The proof of Theorem 1 is complete.

\section{ACKNOWLEDGMENTS}

The author would like to thank the anonymous referee for informing him of a slightly different proof of Theorem 1 which avoids the use of Lemma 2 and a proof of Lemma 2 without detailed case by case analysis. Actually the referee proved that if $G$ has $m$ conjugacy classes of involutions for $m \geq 2$, then $|G|<\left|C_{G}(x)\right|^{3} / m$ for some involution $x$ in $G$ with the use of the Thompson order formula and a counting argument (see Suzuki [13, p. 125-127]). Then he or she applied similar arguments in Lemmas 3, 4 and 5 to prove Theorem 1 by induction. Thus the proof relies less on the classification of finite simple groups.

\section{REFERENCES}

[1] M. Aschbacher and G. Seitz, Involutions in Chevalley groups over fields of even order, Nagoya Math. J. 63 (1976), 1-91. MR0422401 (54:10391)

[2] R. Brauer and K. A. Fowler, On groups of even order, Ann. Math. 62 (1955), 565-583. MR0074414 (17:580e)

[3] N. Burgoyne and C. Williamson, Centralizers of involutions in Chevalley groups of odd characteristic, Mimeographed notes (1972).

[4] N. Chigira, N. Iiyori and H. Yamaki, Non-abelian Sylow subgroups of finite groups of even order, Invent. Math. 139 (2000), 525-539. MR1738059 (2001c:20042)

[5] J. Conway, R. T. Curtis, S. Norton, R. Parker, and R. Wilson, Atlas of finite groups, Clarendon Press, Oxford, 1985. MR827219 (88g:20025)

[6] R. H. Dye, On the conjugacy classes of involutions of the simple orthogonal groups over perfect fields of characteristic two, J. Algebra 18 (1971), 414-425. MR0276366 (43:2113)

[7] R. H. Dye, On the involution classes of the linear groups $G L_{n}(K), S L_{n}(K), P G L_{n}(K)$, $\mathrm{PSL}_{n}(K)$ over fields of characteristic two, Math. Proc. Cambridge Phil. Soc. 72 (1972), 1-6. MR0294519 (45:3589)

[8] R. H. Dye, On the conjugacy classes of involutions of the unitary groups $U_{m}(K), S U_{m}(K)$, $P U_{m}(K), P S U_{m}(K)$ over perfect fields of characteristic 2, J. Algebra 24 (1973), 453-459. MR0308287 (46:7401)

[9] K. Harada and M. Miyamoto, On the order of a group of even order, To appear in J. Algebra.

[10] B. Huppert and N. Blackburn, Finite Groups III, Springer-Verlag, Berlin, 1982. MR662826 (84i:20001b)

[11] N. Iiyori and H. Yamaki, Prime graph components of the simple groups of Lie type over the fields of even characteristic, J. Algebra 155 (1993), 335-343. Corrigenda 181 (1996) 659. MR1212233 (94e:05268)

[12] A. S. Kondrat'ev, Prime graph components of finite simple groups, Math. USSR Sbornik 67 (1990), 235-247. MR1015040 (90h:20018)

[13] M. Suzuki, Group theory II, Springer-Verlag, Berlin, 1986. MR815926 (87e:20001)

[14] J. S. Williams, Prime graph components of finite groups, J. Algebra 69 (1981), 487-513. MR617092 (82j:20054)

Department of Mathematics, Kumamoto University, Kumamoto 860-8555 Japan

E-mail address: yamaki@gpo.kumamoto-u.ac.jp

Current address: JICA, Maipu 1300, Piso 21, C1006ACT Buenos Aires, Argentina

E-mail address: yamaki.hiroyoshi@gmail.com 